\title{
Ripple Mapping-guided Atrial Tachycardia Ablation Following Open-heart Surgery: Interpretation of Reentry Circuits and Selection of Critical Isthmus
}

\author{
Wenzhi Shen ${ }^{1}$, Yu Liu ${ }^{1}$, Jian Bai ${ }^{1}$, Zheng Chen ${ }^{1}$, Xiaohong Li $^{1}$, Rongfang Lan ${ }^{1}$, Zhonglin \\ $\mathrm{Han}^{1}$, Hongsong $\mathrm{Yu}^{1}$, Wenqing $\mathrm{Ji}^{1}$, Biao $\mathrm{Xu}^{1}$, and Wei $\mathrm{Xu}^{1}$ \\ ${ }^{1}$ Nanjing University Medical School Affiliated Nanjing Drum Tower Hospital
}

April 28, 2020

\begin{abstract}
BACKGROUND Ripple mapping (RM) can make the visualization of activation conduction on a 3-dimensinal voltage map. However, its potential advantage in mapping complex atrial tachycardias (ATs) in patients after cardiac surgery has not yet been evaluated. OBJECTIVE To assess the efficacy of ripple mapping for interpreting reentrant circuits and critical isthmus in postoperative ATs. METHODS 24 consecutive patients with a history of open-heart surgery (mean age, 54.5 \pm 12.4 years) underwent high density (HD) RM during ATs with CARTO3v4 CONFIDENSE system. The voltage activation threshold was determined by RM over a bipolar voltage map. Identification of underlying mechanisms and ablation setting were based on RM without reviewing activation mapping. RESULTS A total of 34 ATs (24 spontaneous, 10 induced) were characterized. 32 reentry circuits were successfully mapped (cycle length, $255 \pm 40 \mathrm{~ms}$ ). One focal AT were mapped in the left atrium (LA). Of the 34 ATs, 21 were confirmed by ripple mapping alone (62\%), and $12(32 \%)$ by ripple mapping and entrainment mapping. Of 14 ATs in the left atrium, 8 (57\%) needed entrainment to confirm, compared with $3(15 \%)$ in the right atrium. Primary endpoint after initial ablation set was achieved in 22 of the 24 patients (91.7\%). Both patients were electrically converted to sinus rhythm due to unsuccessful ablation and variable tachycardia cycle length. Conclusion: Ripple mapping precisely delineated reentrant circuits in post cardiac surgery AT resulting in a high success rate of ablation. Entrainment maneuvers remain useful for elucidation of complex AT circuits.
\end{abstract}

\section{Introduction}

Atrial tachycardias (ATs) after open-heart surgery are challenging reentrant arrhythmias, associated with increased mortality and morbidity, and difficult to control with medications(1-3). Underlying arrhythmogenic mechanism is usually considered to be a reentry that involves incisional line, cannulation-related scar and surgical ablation lesions with a resultant sophisticated atrial substrate (4-6).

Conventional 3D activation mapping is a cornerstone for the diagnosis and the ablation of reentrant $\operatorname{ATs}(7)$. However, most studies adopted the combination of activation mapping and entrainment mapping for AT; therefore, the efficacy of activation mapping alone remains unknown and is limited by misannotation of the fractionated potential and arbitrary window of interest, among other factors (8, 9). Ripple mapping (RM) allows the visualization of activation conduction on a 3-dimensinal voltage map thereby addressing the limitations of local activation time (LAT) mapping(10). Several studies have demonstrated that the utility of ripple mapping can improve diagnostic accuracy and ablation outcome compared with LAT mapping approach $(11,12)$. Radiofrequency ablation of atrial tachycardia post cardiac surgery has not been investigated, and is the subject of this study on ripple mapping for interpreting reentrant circuits and critical isthmus.

\section{Methods}




\section{Study Population}

24 consecutive patients post cardiac surgery who were referred to our institution for AT ablation were enrolled prospectively between June 2018 and November 2019. All cases were performed under conscious sedation with continuous intravenous fentanyl. All patients provided written informed content to participate in the study, which was approved by the institutional ethics committee.

\section{Electrophysiological Study}

All antiarrhythmic drugs were discontinued for at least five half-lives, except for amiodarone, which was stopped at least two weeks before the procedure. Transesophageal echocardiography was performed to exclude the presence of thrombus in left atrial appendage prior to the procedure. Electrophysiology study was performed under conscious sedation and local anesthesia. Twelve-lead surface electrocardiograms (ECG) (filtered $0.05-100 \mathrm{~Hz}$ ) and bipolar intracardiac electrograms (filtered $30-250 \mathrm{~Hz}$, sweep speed $100 \mathrm{~mm} / \mathrm{second}$ ) were amplified and displayed on a computer-based digital amplifier/recorder system (Bard Electrophysiology, Lowell, MA, USA).

Following vascular access, a $6 \mathrm{~F}$ quadripolar electrode was placed in the right ventricular apex, and a $6 \mathrm{~F}$ decapolar catheter was positioned in the coronary sinus via the left femoral vein. One $8.5 \mathrm{~F}$ long sheath was advanced into the right atrium through the right femoral vein. A transseptal puncture was performed in the patients with AT originating from left atrium. Regular doses of heparin were administered to maintain an activated clotting time of 300-350 seconds throughout the whole procedure.

\section{Ripple Mapping and Analysis}

Ripple mapping is part of the mapping system module in CARTO3v4 (Biosense Webster). A stable signal from decapolar catheter placed in the coronary sinus was used as reference in all patients. The bipolar electrogram of every point was aligned relative to the reference signal. CARTO3v4 has an automated point collection (ConfiDENSE Continuous mapping). Points were automatically and continuously collected and were only accepted for display on the voltage map after having passed through a series of beat acceptance criteria. At least 1000 points were acquired on a ripple map. To gain satisfactory point collection, the Fill/Color threshold was set to $5 \mathrm{~mm}$. Default threshold of $0.03 \mathrm{mV}$ was considered as noise and not shown, and areas below this threshold were displayed in red and defined as scar. The bars were clipped at $>0.25 \mathrm{mV}$ to display low-voltage bars without being obscured by large bars from areas of healthy tissue(12). Through manual adjustment of voltage scale, the lowest voltage threshold supporting wave fronts of ripple bars was identified when ripple map was played over the voltage map, defined as tissue activation threshold. The ripple map was played as a continuous loop spanning 3 complete cycle lengths of the tachycardia.

The endocardial activation sequence could be appreciated by the movement of each bar relative to its neighbor, creating a ripple effect; wavefront propagation was studied to confirm where it began and ended and whether there was reentrant mechanism. Each wavefront was analyzed to characterize a breakout from local area if reentry circuit was uncertain.

\section{Definition of Reentrant AT and Isthmus Identification}

Reentrant AT was defined as wavefront turning continuously around anatomical obstacle or non-conducting tissue (where head met tail). As previously reported $(13,14)$, reentrant AT was classified into 3 types: Anatomical macro-reentrant AT (AMAT), including peritricuspid AT dependent on the cavo-tricuspid isthmus, perimitral AT dependent on the mitral isthmus, and roof-dependent AT dependent on left atrial roof. Other reentrant ATs were all defined as Non-AMAT. Dual-loop ATs were defined as two dependent circuits sharing one common isthmus, with the entire CL included in each of the circuits.

Anatomical isthmus was reliant on stereotyped isthmuses at the cavo-tricuspid isthmus (CTI), mitral isthmus (MI), and left atrial roof; Non-anatomical isthmuses were characterized by their borders as either "scarscar", or "scar-anatomical". In combination of local potential characteristic, the narrowest channel between 
nonconductive tissues was defined as critical isthmus (CI) in reentrant circuits, where radiofrequency ablation was applied.

\section{LAT Mapping and Entrainment}

During geometry and electroanatomic map acquisition, the window-of-interest (WOI), 0 to $10 \mathrm{~ms}$ shorter than the tachycardia cycle length (TCL), was set in all patients, however, LAT map was not shown on the map before ablation. The entrainment maneuvers (EM) were performed to diagnose AT if initial ablation failed to terminate AT or if AT diagnosis remained uncertain based on the ripple map.

\section{Ablation Settings and Endpoints}

After the critical isthmus or focal origin was identified, power controlled (30-40 W) radiofrequency energy was applied (Stocker 70 RF generator; Biosense Webster) through an irrigated ablation catheter (Thermocool SmartTouch, Biosense Webster), with a cut-off temperature of $43^{\circ} \mathrm{C}$. Catheter ablation was performed using contact force-sensing technology for all patients. For every linear lesion set, bidirectional conduction block was attempted by pacing from both sides of the intended line. PVs were isolated or re-isolated in cases of PV reconduction when needed. The primary endpoint was AT termination with the first ablation set. The first ablation delivery included all ablation lesions targeting the AT based on the studied ripple map.

\section{Statistical Analysis}

Continuous variables are expressed as mean \pm SD and were compared using the t test or the Mann-WhitneyWilcoxon test. Categorical variables are expressed as absolute numbers and proportions and were compared using the chi-squared test or Fisher's exact test. All data analyses were performed with the SPSS system for statistics. Pز0.05 was considered significant.

\section{Results}

\section{Patient Population}

Between June 2018 and December 2019, 24 patients with documented AT after heart surgery (14 females; mean age, 54.5+-12.4years) underwent catheter ablation at our center. Baseline characteristics of the patients are shown in Table 1. All patients had been treated with at least one antiarrhythmic drug and had undergone one or more electric cardioversions prior to catheter ablation procedure. Cardiac surgery had taken place between 2017 and 2019, an average of 20.9+-9.7months before the index ablation. Mitral replacement and concomitant Cox-MAZE was performed in 12 of 24 patients, sole mitral replacement in 5 patients, congenital heart disease repair in 7 patients. Pacemaker was implanted in 2 patients. Mean left ventricular ejection fraction was $53.3 \%+-10.5 \%$, and mean left atrial diameter was $48+-4.7 \mathrm{~mm}$.

\section{Electrophysiological Data}

A total of 34 ATs was documented in 24 patients (1.5+-0.6 ATs per patient, range 1-3). Twenty-four of these tachycardias were present spontaneously at the onset of the electrophysiology study, while the remaining ATs were induced by atrial stimulation or catheter manipulation, with the use of isoproterenol if necessary. The mean AT cycle length was 255+-40ms. High-density electroanatomical maps were created in all patients. The automated point collection facility (ConfiDENSE) was performed in 20 patients with the rest collected point by point (median, 3794+-1096, range 1058-5120). Details are provided in Table 2 . The underlying mechanism was identified as anatomical macroreentrant in 20 ATs (60.7\%), non-anatomical macroreentrant in $12(36.3 \%)$, and 1 focal $(3 \%)$. Cycle length was similar between anatomical and nonanatomical macroreentrant AT $(253+-35$ vs. $257+-49 \mathrm{~ms}, \mathrm{P}=0.61)$. Overall, the chamber of origin was right atrium in 12 patients (50\%), the left atrium in 8 patients (33\%) and biatrial in 4 patients $(17 \%)$.

\section{Reentrant Circuit and Critical Isthmus}

In these patients with AT, the more frequent locations of critical isthmus were CTI-dependent (n=11), free wall of right atrium $(n=5)$, posterior wall of right atrium $(n=3)$, mitral isthmus $(n=3)$, and left atrium roof $(n=3)$ (Table 2). Twenty-one ATs were diagnosed by ripple mapping only (62\%), compared with 12 
(35\%) by ripple mapping and EM. Of 20 anatomical macroreentrant ATs, 6 ATs needed to be confirmed by entrainment mapping. However, 6 of 13 non-anatomical macroreentrant ATs needed EM to make correct diagnosis. Of 14 ATs in the left atrium, 8 (57\%) needed entrainment to confirm, compared with $3(15 \%)$ in the right atrium.

The conduction channel was confirmed easily on the ripple map. Figure 1 clearly illustrates that critical isthmus after adjustment of voltage threshold is located between nonconduction tissues in posterior right atrium for which ablation application was delivered to terminate AT. Left AT frequently occurred in the patients with prior Maze procedure. An example of LA complex AT in Figure 2 clearly demonstrated a circuit around a small area of nonconduction tissue on the anterior wall, without ripple wavefronts through it. A narrow and slowly conducting isthmus between the "scar island" and right superior PV, and ablation line on conduction channel terminated the tachycardia. Four double-loop macroreentries (15\%) were diagnosed (3 in right atrium, 1 in left atrium), one of which was peri-tricuspid and incisional line reentry. Ablation application was first delivered on the line between inferior vena cava and incisional lesion, followed by CTI line ablation (Figure 3).

\section{Ablation Outcome}

Acute termination of AT, as defined in the Methods section, was achieved in 22 (91.7\%) of 24 patients with the first ablation set. The ablation of AT guided by ripple mapping alone was acutely successful in 13 patients (59\%). In one patient, the mechanism could not be determined because of cycle length instability impeded complete mapping, and therefore ripple mapping and EM were not performed. Mitral isthmus-dependent AT, determined by ripple mapping and EM, could not be terminated by conventional mitral isthmus and anterior wall line ablation in one case. These two patients were converted electrically to sinus rhythm, and preventive ablation was performed based on voltage mapping.

\section{Discussion}

\section{Major Findings}

The present study demonstrated that: (1) ripple mapping can precisely delineate reentrant circuit of complex AT after open-heart surgery; (2) individual adjustment of voltage threshold can easily help to display critical isthmus and guide ablation lesion set; and (3) addition of entrainment maneuvers to ripple mapping significantly improved diagnostic accuracy of AT, especially in complex left atrium AT after Maze procedure.

\section{Advantages of ripple mapping for Post-surgical AT Substrate}

AT in long-term survivors post open-heart surgery is a challenging clinical problem(2). The most common mechanism of these arrhythmias is macroreentry, however, they can also be secondary to localized reentry or microreentry involving surgical incisional line or surgery-related scar. Differentiation of scar tissue from active tissue is important to locate critical isthmus and improve success rate of ablation. There is no consensus on atrial voltage threshold to define true atrial scar, and there are scarce data on normal voltage standard for healthy atrium. Variation in bipolar voltage amplitude usually is accompanied by changes in atrial activation sequence due to different heart rhythm or anisotropic myocardial characteristics; such variations may be important in the low voltage area that supports re-entry. At procedure onset, presetting voltage threshold is arbitrary which can mislead interpretation of LAT map owing to interpolation algorithms in unmapped regions. Ripple mapping provides a means of discriminating inactive tissue, through which activation wavefronts of the ripple bars cannot propagate, defined as voltage thresholding (namely tissue activation threshold). Continuous manual adjustment of voltage threshold helps to display critical electrophysiological isthmus (location and width) supporting AT maintenance (Figure 1), allowing the ablation set to be minimized and individualized, in contrast to the case with empirical ablation lesions in the conventional sites.

Conventional LAT mapping is an established tool to identify the mechanisms of AT as well as to guide ablation. However, activation mapping of complex scar-related AT is frequently difficult to interpret due to arbitrary WOI setting, incorrect annotation of the fractionated potential and inappropriate interpolation 
between unmapped sites(8). Several studies have reported macro pseudo reentrant circuit in post-surgical ATs because of focal source or a reentrant AT with slow conduction areas. The example in Figure 4 is a case where pseudo reentrant circuit in the posterior RA was diagnosis based on the LAT map. However, the ripple map suggested that AT originated from left atrium. Finally, perimitral flutter was confirmed. A recent study by Xue et al. on patients with post-surgical AT reported an incidence of macro pseudo reentry approaches of up to $15.7 \%(15)$. It is well known that there are more slowly conduction areas and conduction bock lines in patients who had undergone surgical repair of complex chronic heart disease or Maze procedure, which may result in higher pseudo reentry. Luther et al. also reported localized pseudo reentry in complex iatrogenic AT with ultra-high-resolution mapping in up to $86 \%$ of cases in small rotatory activation covering full cycle length map(16). RM is a novel approach to display wave front propagation, which does not require annotation of EGM activation time or WOI setting. In our study, diagnostic yield of $63 \%$ in AT mechanisms was achieved with sole ripple mapping, as confirmed by ablation outcomes based on ripple maps. A recent randomized controlled study showed that only $44 \%$ of ATs were acutely terminated with first ablation application based on conventional LAT mapping without entrainment maneuvers(17).

\section{Additional Value of Entrainment Mapping in Complex AT}

Entrainment mapping is a pivotal electrophysiological approach in identifying the reentrant mechanism as well as localizing the critical isthmus for ablation(18). With the evolution of high-density LAT mapping, not only simple reentrant circuits but also complex circuits can be confirmed particularly in the setting of abnormal atrial anatomy, scarring area and multiple circuits, which may reduce dependence on EM. However, because of the inherent limitations of activation mapping mentioned above, EM still has an irreplaceable role in identifying mechanisms of arrhythmias. A study on complex left AT by Strisciuglio et al. showed that EM with concomitant high-density left AT mapping significantly improved diagnostic accuracy compared with high-density left AT mapping alone, particularly in microreentries(19). Another study comparing highresolution mapping with EM in interpretation of right atrial flutter circuits reported a $100 \%$ correlation between activation mapping and EM for CTI upper loop reentrant circuits, and of only $30 \%$ for lower loop reentrant circuits; there was no correlation for lateral wall reentry, and EM confirmed only 17 of the 27 (63\%) circuits visualized on the LAT map as indeed being active reentry(20). Our study also demonstrated that ripple mapping combined with EM significantly improved diagnostic yield of AT mechanisms compared with ripple mapping alone, especially in patients with Cox-MAZE procedure. A randomized clinical study by Luther et al. reported that combination of EM and ripple mapping significantly increased success rate of ablation, with patients in ripple mapping group undergoing less EM than those in LAT group(17).

Although EM is not without its limitations, including change or termination of AT, noncapture, and pacing latency in areas of low voltage, EM remains a powerful tool to confirm visual reentrant circuit and corroborate interpretation of eletroanatomical map obtained by high-resolution mapping, either with ripple mapping or LAT mapping.

\section{Study Limitations}

Interpretation and generalizability of findings in this study are limited by the study's single center design with a relatively small sample size. Although all patients were enrolled prospectively, patient characteristics differed among individuals. Eleven patients underwent MV replacement and Maze procedure, which leads to more complex scar regions and conduction block lines with resultant complicated reentrant circuits, while the other patients underwent relatively simple surgical repair due to atrial or ventricular septal defect. Comparison between ripple mapping and activation mapping was not performed because activation map was not shown during the procedure. Absolute value of the activation threshold determined by voltage measurement methodology is dependent on the recording technique and background noise of the mapping system, which will influence the resolution of ripple mapping. Besides, ablation catheter was used to acquire points for eletroanatomical geometry in 4 patients. It is unclear how much impact catheter type has on the activation threshold and ablation outcome in the present study.

\section{Conclusion}


Our series of complex reentrant circuits in postsurgical ATs highlight the importance of high-density ripple mapping to facilitate the delineation of circuits and identification of critical isthmus in the scarring atrium and allow successful ablation with minimal lesion set. EM remains a useful electrophysiological technique in improving diagnostic confidence level and ameliorating outcomes of ablation in the context of high-density 3D mapping system.

\section{Reference}

1. Creswell LL, Schuessler RB, Rosenbloom M, Cox JL. Hazards of postoperative atrial arrhythmias. Ann Thorac Surg. 1993;56(3):539-49.

2. Avila P, Oliver JM, Gallego P, Gonzalez-Garcia A, Rodriguez-Puras MJ, Cambronero E, et al. Natural History and Clinical Predictors of Atrial Tachycardia in Adults With Congenital Heart Disease. Circ Arrhythm Electrophysiol. 2017;10(9);

3. Wu J, Deisenhofer I, Ammar S, Fichtner S, Reents T, Zhu P, et al. Acute and long-term outcome after catheter ablation of supraventricular tachycardia in patients after the Mustard or Senning operation for D-transposition of the great arteries. Europace. 2013;15(6):886-91.

4. Walsh EP, Cecchin F. Arrhythmias in adult patients with congenital heart disease. Circulation. 2007;115(4):53445.

5. Nakagawa H, Shah N, Matsudaira K, Overholt E, Chandrasekaran K, Beckman KJ, et al. Characterization of reentrant circuit in macroreentrant right atrial tachycardia after surgical repair of congenital heart disease: isolated channels between scars allow "focal" ablation. Circulation. 2001;103(5):699-709.

6. Pap R, Kohari M, Makai A, Bencsik G, Traykov VB, Gallardo R, et al. Surgical technique and the mechanism of atrial tachycardia late after open heart surgery. J Interv Card Electrophysiol. 2012;35(2):12735.

7. Del Carpio Munoz F, Buescher TL, Asirvatham SJ. Three-dimensional mapping of cardiac arrhythmias: what do the colors really mean? Circ Arrhythm Electrophysiol. 2010;3(6):e6-11.

8. Ju W, Yang B, Chen H, Zhang F, Gu K, Yu J, et al. Mapping of focal atrial tachycardia with an uninterpretable activation map after extensive atrial ablation: tricks and tips. Circ Arrhythm Electrophysiol. 2014;7(4):598-604.

9. Rav-Acha M, Ng CY, Heist EK, Rozen G, Chalhoub F, Kostis WJ, et al. A Novel Annotation Technique During Mapping to Facilitate the Termination of Atrial Tachycardia Following Ablation for Atrial Fibrillation. J Cardiovasc Electrophysiol. 2016;27(11):1274-81.

10. Linton NW, Koa-Wing M, Francis DP, Kojodjojo P, Lim PB, Salukhe TV, et al. Cardiac ripple mapping: a novel three-dimensional visualization method for use with electroanatomic mapping of cardiac arrhythmias. Heart Rhythm. 2009;6(12):1754-62.

11. Luther V, Linton NW, Koa-Wing M, Lim PB, Jamil-Copley S, Qureshi N, et al. A Prospective Study of Ripple Mapping in Atrial Tachycardias: A Novel Approach to Interpreting Activation in Low-Voltage Areas. Circ Arrhythm Electrophysiol. 2016;9(1):e003582.

12. Luther V, Cortez-Dias N, Carpinteiro L, de Sousa J, Balasubramaniam R, Agarwal S, et al. Ripple mapping: Initial multicenter experience of an intuitive approach to overcoming the limitations of 3D activation mapping. J Cardiovasc Electrophysiol. 2017;28(11):1285-94.

13. Jais P, Matsuo S, Knecht S, Weerasooriya R, Hocini M, Sacher F, et al. A deductive mapping strategy for atrial tachycardia following atrial fibrillation ablation: importance of localized reentry. J Cardiovasc Electrophysiol. 2009;20(5):480-91.

14. Takigawa M, Derval N, Frontera A, Martin R, Yamashita S, Cheniti G, et al. Revisiting anatomic macroreentrant tachycardia after atrial fibrillation ablation using ultrahigh-resolution mapping: Implications 
for ablation. Heart Rhythm. 2018;15(3):326-33.

15. Xue Y, Liu Y, Liao H, Zhan X, Fang X, Deng H, et al. Evaluation of Electrophysiological Mechanisms of Post-Surgical Atrial Tachycardias Using an Automated Ultra-High-Density Mapping System. JACC Clin Electrophysiol. 2018;4(11):1460-70.

16. Luther V, Sikkel M, Bennett N, Guerrero F, Leong K, Qureshi N, et al. Visualizing Localized Reentry With Ultra-High Density Mapping in Iatrogenic Atrial Tachycardia: Beware Pseudo-Reentry. Circ Arrhythm Electrophysiol. 2017;10(4).

17. Luther V, Agarwal S, Chow A, Koa-Wing M, Cortez-Dias N, Carpinteiro L, et al. Ripple-AT Study: A Multicenter and Randomized Study Comparing 3D Mapping Techniques During Atrial Tachycardia Ablations. Circ Arrhythm Electrophysiol. 2019;12(8):e007394.

18. Kalman JM, Olgin JE, Saxon LA, Fisher WG, Lee RJ, Lesh MD. Activation and entrainment mapping defines the tricuspid annulus as the anterior barrier in typical atrial flutter. Circulation. 1996;94(3):398-406.

19. Strisciuglio T, Vandersickel N, Lorenzo G, Van Nieuwenhuyse E, El Haddad M, De Pooter J, et al. Prospective evaluation of entrainment mapping as an adjunct to new-generation high-density activation mapping systems of left atrial tachycardias. Heart Rhythm. 2020;17(2):211-9.

20. Pathik B, Lee G, Nalliah C, Joseph S, Morton JB, Sparks PB, et al. Entrainment and high-density threedimensional mapping in right atrial macroreentry provide critical complementary information: Entrainment may unmask "visual reentry" as passive. Heart Rhythm. 2017;14(10):1541-9.

\section{Figure legends}

\section{Figure 1 Nonconducting Tissue Identification}

A patient with sustained AT after Fanton Modification. (Movie I and Movie II in the Data Supplement). A: Bipolar voltage threshold is set arbitrarily 0.5 to $0.5 \mathrm{mV}$, with red representing voltage $<0.5 \mathrm{mV}$ and purple representing $>0.5 \mathrm{mV}$. Ripple bars are seen to propagate in the red area on the map (yellow circle). B: After adjustment of voltage threshold, the area with propagating ripple bars appears as purple (yellow circle), representing conductive tissue. C, D: At this voltage setting, the ripple map of the RA during posterior scar-related flutter in this case that clearly delineated the critical isthmus located between the posterior scar and IVC. RA: right atrium; SVC: superior vena cava; CS: coronary sinus; IVC: inferior vena cava.

\section{Figure 2 Identification of the Critical Isthmus on Ripple Map}

Patient with left atrial tachycardia after MV replacement concomitantly with Maze procedure. (Movie III and Movie IV in the Data Supplement). A: The activation threshold is progressively reduced until 0.07 $\mathrm{mV}$-no ripple bars were seen in areas colored red. The PentaRay catheter was placed on the non-active area, with corresponding electrograms (EGMs) covered the atrial tachycardia cycle length. B, C: The block line existed between RSPV and non-active area, and slow area was present between the block line and the scar island. D: Based on the ripple map and local EGMs, the localized reentrant circuit turned around non-conductive area (marked out by yellow arrows), with the critical isthmus located between non-active area and the block line (black dotted line). Atrial tachycardia was terminated by ablating the conduction gap (wavy line). RSPV: right superior pulmonary vein; LSPV: left superior pulmonary vein; MV: mitral valve.

\section{Figure 3 Figure-8 Macro Re-Entrant AT in a Patient with ASD Repair}

Dual-loop reentry with a clockwise $(\mathrm{CW})$ free wall loop and a counterclockwise $(\mathrm{CCW})$ peritricuspid flutter (AT TCL=266ms). A, B: As shown in Movie V in the Data Supplement, ripple map demonstrated a figure of 8 circuit (marked out by yellow arrows). Ablation application was first delivered on the line between IVC and incisional lesion (black dotted line), resulting in TCL converted to 288ms. C, D: After remapping the RA, the mechanism of AT is peritricuspid flutter (See Movie VI in the Data Supplement). CTI ablation 
terminated the flutter. ASD: atrial septal defect; AT: atrial tachycardia; TCL: tachycardia cycle length; Other abbreviations as in Figure 1.

\section{Figure 4 Pseudo Reentrant Circuit in a Patient with Previous MV Replacement}

The block line and scar create the false diagnosis of small reentrant circuit. Atrial tachycardia was mapped in the RA in Patient with a prior MV replacement (AT TCL=204ms). A: LAT map of the RA during AT demonstrating "chaotic activation". B: Reannotating the points by moving the window of interest resulted in the appearance of the early-meets-late pattern in the posterior RA. C: There was a non-conducting area on the bipolar map in the posterior RA. Based on the early-meets-late pattern around the posterior RA corresponding to the area of low voltage, small loop reentry was considered to be the mechanism of AT. D, E, F: There was no evidence of wavefront rotation on the ripple map, rather splitting of wavefronts on either side of the low voltage area (See Movie VII in the Data Supplement). The ripple map demonstrated that a line of conduction block prevented small loop reentry around this pitch of scar, evident by a line of double potentials long it (black dotted line). The pattern of activation is represented by yellow arrow line. Finally, perimitral circuit was confirmed, and ablation of anterior line in the LA terminated AT in this case. MV: mitral valve; AT: atrial tachycardia; LA: left atrium; TCL: tachycardia cycle length; abbreviations as in Figure 1.

\begin{tabular}{lll}
\hline Characteristic & Value & Value \\
\hline Age (yrs) & Age (yrs) & $54.5 \pm 12.4$ \\
Sex (female, \%) & Sex (female, \%) & $14(58.3 \%)$ \\
Cardiac surgery & Cardiac surgery & \\
Atrial septal defect repair & Atrial septal defect repair & $6(25 \%)$ \\
MV replacement/repair & MV replacement/repair & $5(20.8 \%)$ \\
MV replacement/repair + MAZE & MV replacement/repair + MAZE & $12(50 \%)$ \\
IV & IV & $1(4.2 \%)$ \\
Fanton Modification & Fanton Modification & $11(45.8 \%)$ \\
Hypertension & Hypertension & $6(25 \%)$ \\
Diabetes mellitus & Diabetes mellitus & $1(4 \%)$ \\
Stroke & Stroke & $1.5 \pm 1.3$ \\
CHA2DS2-VASc & CHA2DS2-VASc & $11(45.8 \%)$ \\
OAC (warfarin, \%) & OAC (warfarin, \%) & $15 \pm 7$ \\
Months since surgery & Months since surgery & $48 \pm 4.7$ \\
LAD (mm) & LAD (mm) & $53.3 \pm 10.5$ \\
LVEF (\%) & LVEF (\%) & $2(8 \%)$ \\
Pacemaker implantation & Pacemaker implantation & \\
Anti-arrhythmia medications & Anti-arrhythmia medications & $19(79.1 \%)$ \\
$\beta$-blocker & $\beta$-blocker & $10(41.6 \%)$ \\
Calcium-channel blockers & Calcium-channel blockers & $8(33.3 \%)$ \\
Class I or III & Class I or III &
\end{tabular}

Table 1 Baseline Demographic

Values are n (\%) or mean \pm SEM. MV: mitral valve; OAC: oral anticoagulant; LAD: left atrium diameter; LVEF: left ventricular ejection fraction.

Table 2 Electrophysiological Details of ATs 


\begin{tabular}{|c|c|c|c|c|c|c|c|c|c|}
\hline Pt. No. & $\begin{array}{l}\text { Mapping } \\
\text { Data }\end{array}$ & $\begin{array}{l}\text { Mapping } \\
\text { Data }\end{array}$ & $\begin{array}{l}\text { Mapping } \\
\text { Data }\end{array}$ & $\begin{array}{l}\text { ATCL } \\
(\mathrm{ms})\end{array}$ & EM & $\begin{array}{l}\text { Critical } \\
\text { Isthmus } \\
\text { Location }\end{array}$ & $\begin{array}{l}\text { Isthmus } \\
\text { Voltage } \\
\text { Threshold }\end{array}$ & AT Type & $\begin{array}{l}\mathrm{Ac} \\
\mathrm{Su}\end{array}$ \\
\hline & $\begin{array}{l}\text { Mapping } \\
\text { Catheter }\end{array}$ & $\begin{array}{l}\text { Target } \\
\text { Chamber }\end{array}$ & Points & & & & & & \\
\hline 1 & PentaRay & LA & 3987 & 206 & No & MI & 0.21 & AMAT & $\mathrm{Y}$ \\
\hline 2 & PentaRay & LA & 4432 & 250 & Yes & AW & 0.07 & NAMAT & Y \\
\hline \multirow[t]{2}{*}{3} & PentaRay & RA & 4909 & 220 & No & CTI & 0.09 & AMAT & Y \\
\hline & PentaRay & RA & 3890 & 230 & No & CTI & 0.33 & NAMAT & Y \\
\hline 4 & PentaRay & RA & 4210 & 245 & No & CTI & 0.04 & NAMAT & Y \\
\hline 5 & PentaRay & RA & 3508 & 255 & No & FW & 0.35 & AMAT & Y \\
\hline 6 & $\begin{array}{l}\text { Ablation } \\
\text { Catheter }\end{array}$ & RA & 1252 & 230 & No & CTI & 0.11 & AMAT & Y \\
\hline 7 & PentaRay & LA & 4432 & 275 & Yes & Roof & 0.09 & AMAT & $\mathrm{N}$ \\
\hline 8 & PentaRay & LA & 4930 & 248 & Yes & PW & 0.06 & NAMAT & Y \\
\hline I9 & PentaRay & RA & 3495 & 285 & No & PW & 0.4 & NAMAT & Y \\
\hline \multirow[t]{2}{*}{10} & PentaRay & RA & 4439 & 300 & No & CTI & 0.55 & AMAT & Y \\
\hline & PentaRay & RA & 3763 & 270 & Yes & FW & 0.09 & AMAT & Y \\
\hline 11 & PentaRay & RA & 4190 & 225 & No & CTI & 0.19 & AMAT & Y \\
\hline 12 & $\begin{array}{l}\text { Ablation } \\
\text { Catheter }\end{array}$ & LA & 1359 & None & No & None & None & None & $\mathrm{N}$ \\
\hline 13 & PentaRay & $\mathrm{RA}$ & 4568 & 240 & No & CTI & 0.07 & AMAT & $\mathrm{Y}$ \\
\hline \multirow[t]{2}{*}{14} & PentaRay & RA & 3210 & 210 & Yes & PW & 0.11 & AMAT & $\mathrm{Y}$ \\
\hline & PentaRay & LA & 4865 & 230 & Yes & MI & 0.05 & AMAT & $\mathrm{Y}$ \\
\hline \multirow[t]{2}{*}{15} & PentaRay & $\mathrm{RA}$ & 3389 & 220 & Yes & FW & 0.25 & AMAT & $\mathrm{Y}$ \\
\hline & PentaRay & $\mathrm{RA}$ & 4780 & 225 & Yes & PW & 0.09 & NAMAT & $\mathrm{Y}$ \\
\hline 16 & PentaRay & RA & 4135 & 290 & No & CTI & 0.45 & AMAT & $\mathrm{Y}$ \\
\hline \multirow[t]{2}{*}{17} & PentaRay & LA & 3954 & 210 & Yes & AW & 0.07 & NAMAT & $\mathrm{Y}$ \\
\hline & PentaRay & LA & 4095 & 320 & No & Roof & 0.06 & NAMAT & $\mathrm{Y}$ \\
\hline 18 & $\begin{array}{l}\text { Ablation } \\
\text { Catheter }\end{array}$ & RA & 1156 & 220 & No & CTI & 0.25 & AMAT & $\mathrm{Y}$ \\
\hline 19 & PentaRay & LA & 5120 & 240 & Yes & Septum & 0.08 & NAMAT & $\mathrm{Y}$ \\
\hline \multirow[t]{3}{*}{20} & PentaRay & LA & 4835 & 250 & No & PW & 0.3 & NAMAT & $\mathrm{Y}$ \\
\hline & PentaRay & RA & 3995 & 330 & No & $\mathrm{FW}$ & 0.25 & AMAT & $\mathrm{Y}$ \\
\hline & PentaRay & LA & 4698 & 280 & No & MI & 0.13 & AMAT & $\mathrm{Y}$ \\
\hline 21 & $\begin{array}{l}\text { Ablation } \\
\text { Catheter }\end{array}$ & LA & 1058 & 295 & Yes & Roof & 0.08 & AMAT & $\mathrm{Y}$ \\
\hline 22 & PentaRay & RA & 3560 & 224 & No & CTI & 0.35 & AMAT & $\mathrm{Y}$ \\
\hline \multirow[t]{2}{*}{23} & PentaRay & LA & 4250 & 380 & No & AW & 0.15 & NAMAT & $\mathrm{Y}$ \\
\hline & PentaRay & RA & 4128 & 288 & No & FW & 0.09 & AMAT & $\mathrm{Y}$ \\
\hline \multirow[t]{3}{*}{24} & PentaRay & RA & 3350 & 210 & No & Septum & 0.5 & NAMAT & $\mathrm{Y}$ \\
\hline & PentaRay & RA & 2970 & 260 & No & CTI & 0.13 & AMAT & $\mathrm{Y}$ \\
\hline & PentaRay & LA & 4109 & 320 & No & None & None & Focal & $\mathrm{Y}$ \\
\hline
\end{tabular}

Pt: patient; ATs: atrial tachycardias; LA: left atrium; RA: right atrium; ATCL: atrial tachycardia cycle length; EM: entrainment maneuver; MI: mitral isthmus; AW: anterior wall; CTI: cavo-tricuspid isthmus; FW: free wall; PW: posterior wall; AMAT: anatomical macroreentrant atrial tachycardia; NAMAT: nonanatomical macroreentrant atrial tachycardia. 

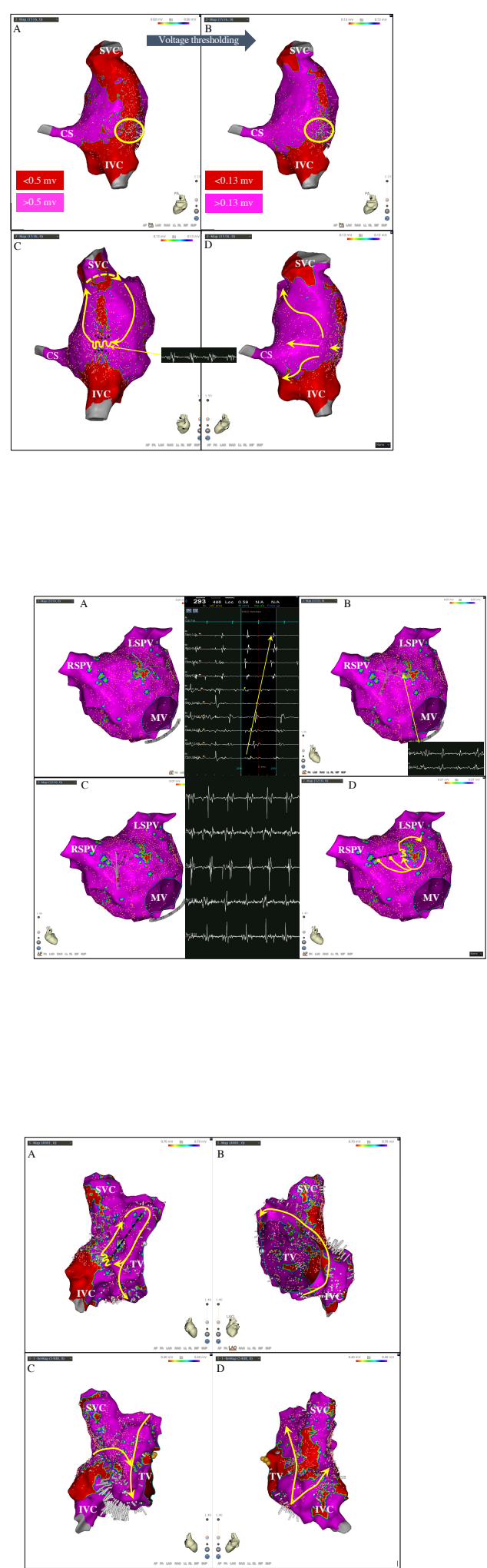


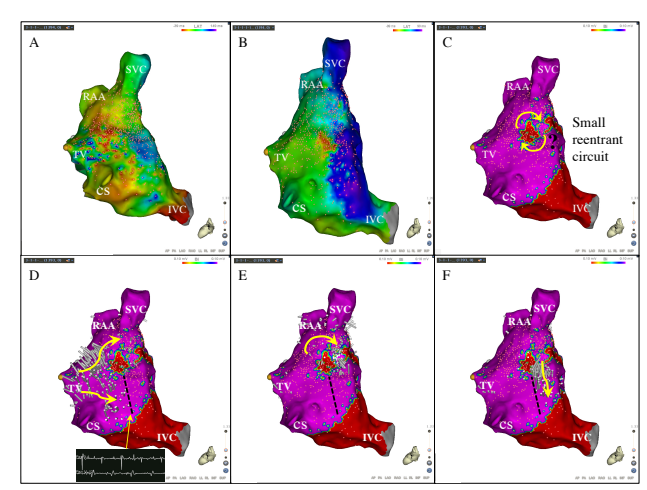

